\title{
Lifetime Determination for Delay Tolerant Communications in Sparse Vehicular Networks
}

\author{
Kadri Kaan Sevimli, Mujdat Soyturk \\ Department of Computer Engineering, Turkish Naval Academy \\ Tuzla, Istanbul, 34942, Turkey \\ ksevimli@dho.edu.tr \\ msoyturk@dho.edu.tr
}

\begin{abstract}
Classical ad-hoc network algorithms become impractical for Vehicular Ad Hoc Networks when density of vehicles is low in rural areas and during the periods when the vehicle traffic is low in cities (such as at night). End-to-end connectivity requirement of these algorithms causes deflection. Connections get broken when a suitable network element isn't available for data transfer. This problem which occurs in low dense partially connected Vehicular Ad hoc Networks can be solved by using delay tolerant approaches in routing algorithms. By using the store-carry-forward method, data is carried by network elements until a suitable network element is found. By this way, network connectivity is provided in a delayed manner between momentarily disconnected vehicles. In this paper, the impact of delayed transfer of data to an end-to-end transfer and the impact of data lifetime to the network performance are examined. Considering the speed of vehicles and the desired throughput, lifetime of data is determined according to the network connectivity. The results of this study contribute to the fulfilment of the Quality of Service (QoS) requirements in Vehicular Ad Hoc Network applications.
\end{abstract}

\section{INTRODUCTION}

Studies on Vehicular Networks has expedited in recent years and this technology become applicable as a result of advances in wireless communications. Though the initial studies oriented to safety, its applicability to other areas such as business, entertainment, driving assist and public services, studies enlarged to comprise these new areas.

Vehicular Ad Hoc Networks use the DSRC (Dedicated Short Range Communications) [1] standard presenting high data rate $(6-27 \mathrm{Mbps})$ which has a bandwidth of $75 \mathrm{MHz}$ and band of $5.9 \mathrm{GHz}$. With DSRC, vehicles on the road broadcast the routine traffic message which includes location, time, direction, speed and traffic condition information. Additionally, emergency messages (e.g. accident, emergency brake) are sent on emergency conditions. While the routine messages are single-hop beaconing messages, emergency messages can be sent over multiple-hop paths.

The main advantage of Vehicular Ad Hoc Networks is their applicability as an alternative communication network in case of catastrophes such as earthquake, flood etc. due to adhoc nature which does not require network infrastructure.
Vehicular Ad Hoc Networks differ from Mobile Ad Hoc Networks (MANET) with respect to speed of nodes and dependency to roads on node movements. Nodes move at high speed on the same or opposite directions while the speed may change in time. High speed causes connection/route breakages on transmissions. Routing algorithms proposed for MANETs become impractical due to reasons given above. Moreover, node density in VANETs may change very frequently in time and space while it is not frequently expected to be in MANETs. Additionally, Quality of Service (QoS) requirements in VANETs differ due to their varying application areas.

Network density which changes according to the number of nodes and their speeds affects the performance of routing algorithms and thus performance of the network. Examples to varying densities are urban areas with high traffic and highways/rural areas with low traffic. Node density changes not only according to topological areas but also to time. While traffic is heavy in highways and urban areas during work hours (and at suitable density for emergency message transfer over multi-hop paths), there are less vehicles on those roads at nights which increase the interruptions and route breakages in data transfer. While high node density causes broadcast storm problem [2], low density has negative impact on network connectivity. In partially connected networks, emergency messaging becomes impossible in real time. Message can be sent in delay tolerant fashion by store-carryforward method. Message is delayed at nodes until a suitable node is found to forward the message. Delaying packets at nodes increases end-to-end delay and makes usual lifetime usage impractical. Lifetime of the packets needs to be determined considering the delay time at nodes. Short lifetime causes packet losses and long lifetime increases load at nodes due to increased number of buffered packets. Finite buffer size limits the lifetime of packets at a maximum. Moreover, mobility, network connectivity depending on node density and range, and Quality of Service requirements of applications affects lifetime determination of the packets to be sent in such sparse networks. Determined lifetime affects the performance of the network. 
In this paper, we study to determine how long a packet can be delayed to increase the networks performance in terms of packet delivery ratio and also present the effects of network density and nodes' mobility in determination of packet lifetime. As far as we know, this is the first study in determination of effective lifetime for sparse vehicular networks.

Paper is organized as follows. Studies and related work about sparse vehicular networks is presented in the second section. In the third section, the effect of lifetime to network performance is presented. Simulation and results presented in the fourth section. Conclusion is given in the last section.

\section{RELATED WORKS}

Classical Ad hoc routing algorithms (such as DSR, AODV etc.) becomes inadequate and impractical for Vehicular Ad Hoc Networks when the network density is low. Algorithms proposed for Vehicular Ad hoc Networks are given in [3], [4]. The simplest approach that can be used for low network density is spreading the message through the network to make sure that it reaches to the destination. Epidemic Routing Algorithm [5] uses this method; data packets are spread within the network as in the flooding algorithm. Therefore, this approach has all unfavorable features of this algorithm. Dissemination of packets through the network is restrained by Spray\&Wait [6] and MoVe [7] algorithms and unnecessary packets in the network are reduced in these algorithms. MoVe algorithm allows nodes to forward packets to vehicles whose direction is towards the destination while Spray\&Wait algorithm sends packets to a certain number of vehicles chosen at random. Aim of all these algorithms is utilizing simultaneous multiple paths to make sure the message reach to the destination. With these algorithms, however, it is not possible to communicate between disjoint clusters in a partially connected network. It is impossible to find a path between pairs of nodes those are in disjoint clusters.

Predictive Graph Relay (PGR) [8], Delay-Bounded Greedy Forwarding (D-Greedy), and Delay-Bounded Minimum Cost Forwarding (D-MinCost) [9] are the geographical based algorithms those aim to transfer the packet by delaying in low density networks. Though the data delivery rate is enhanced in these algorithms, they neither address the issues about delay at nodes and packet lifetime nor make an evaluation about these issues. One early study about end-to-end delay in Delay Tolerant Networks is presented in [10]. In their study, which is based on Mobility Aware Routing Protocol and Mobility Dissemination Protocol (MARP/MDP) [11] for airborne networks, authors try to predict the minimum end-to-end delay and obtain the corresponding path for airborne networks. However, they do not address the issues related with carry time and lifetime. Moreover, with regard to different network connectivity values and speed of vehicles, the time needed to transfer the data packets and lifetime of packets to remain in the network will change. This study is a first one to address these issues and to determine lifetime in sparse vehicular networks.

\section{THE EFFECT OF DATA LIFETIME TO NETWORK PERFORMANCE IN DELAY TOLERANT NETWORK}

Vehicles in sparse networks remains in clusters as partially connected when the full network connectivity is not provided depending on node density and speed of nodes. This affects the data transfer at an instant time causing route breakages and packet losses in end-to-end connections. Storecarry-forward method can be used in order to minimize the packet losses in route breakages. In this mechanism, in case of no available neighbor nodes to forward the packet, the vehicle stores the packet and carries until it finds a vehicle/path to send and then forwards the packet. This method is very effective because the vehicles' movements at different directions and varying speeds cause members of the clusters in a partially connected network interchange their member(s) and hence clusters are formed with these new member(s). Clusters are partitioned and are reformed continually. By this way, even if there is not an end-to-end path at an instant time, due to continuous topology change in the network, the message will arrive to the destination in delayed time. This end-to-end delay can be considerably high depending on the network connectivity, speed of nodes and some other parameters.

Key consideration for this method is determination of waiting times, in other words delaying times at nodes; how long the message will be carried by vehicles. Depending on the carry time of the message, successful delivery probability of the message changes. Packet is dropped or is carried by the vehicles according to determined carry time. Depending on this carry time, lifetime of the packets in the networks varies, but using the packet lifetime infinite will cause the packets accumulate at nodes forming a mass. This will cause overhead in the network requiring infinite buffer size at nodes, which will result unbounded delay and congestion in the network. Similarly, even in case the lifetime is determined appropriately, high buffer sizes can cause packet losses due to increased waiting time in the buffer and low buffer size can cause packet losses due to insufficient buffer size to store packets.

Another key point that needs to be considered is the Quality of Service requirements of the applications. Packets should be delivered to intended destinations within a certain time and an acceptable packet loss rate. Therefore, packet carry time at nodes and lifetime of the packets within the network should also be determined considering these metrics. Since variable network density in partially connected networks affects the probability of vehicle interchange within clusters, the successful packet delivery ratio is affected from packet lifetime that is determined with respect to network density. Likewise, as the distance taken by the vehicles changes according to the vehicles' speed, vehicles' packet carry time required to find an appropriate path to the destination is affected from vehicles' speed.

Studies in the literature generally address the issues related to enhance the network performance and to increase the network connectivity. However, in these studies their impact on the lifetime has not been mentioned. There is a 
need to determine efficient packet lifetime to maximize the network performance in terms of packet delivery ratio and Quality of Service requirements of applications. In this study, we address these issues. Packet carry time at nodes and packet lifetime within the network are examined with respect to network connectivity and vehicle speed. Their effect on successful packet delivery ratio is also examined.

\section{SIMULATIONS AND RESULTS}

In this section, we present the experimental results comparing the performance of Delay Tolerant Networks (DTN) with Non-Delay Tolerant Networks (NDTN). Parameters related with the simulations are given in the next subsection. Simulations are conducted on networks with different densities. Performance comparisons include effects of lifetime and vehicles' speed to packet delivery ratio for different partially connected networks.

\section{A. Simulation Parameters}

In simulations, Manhattan Grid Topology and Mobility Model [12] are used for the Vehicular Ad hoc Network topology and traffic model. Fig. 1 shows the simulated network topology at an instant time in an urban area. Parameters values are determined to be similar in other related studies [13].

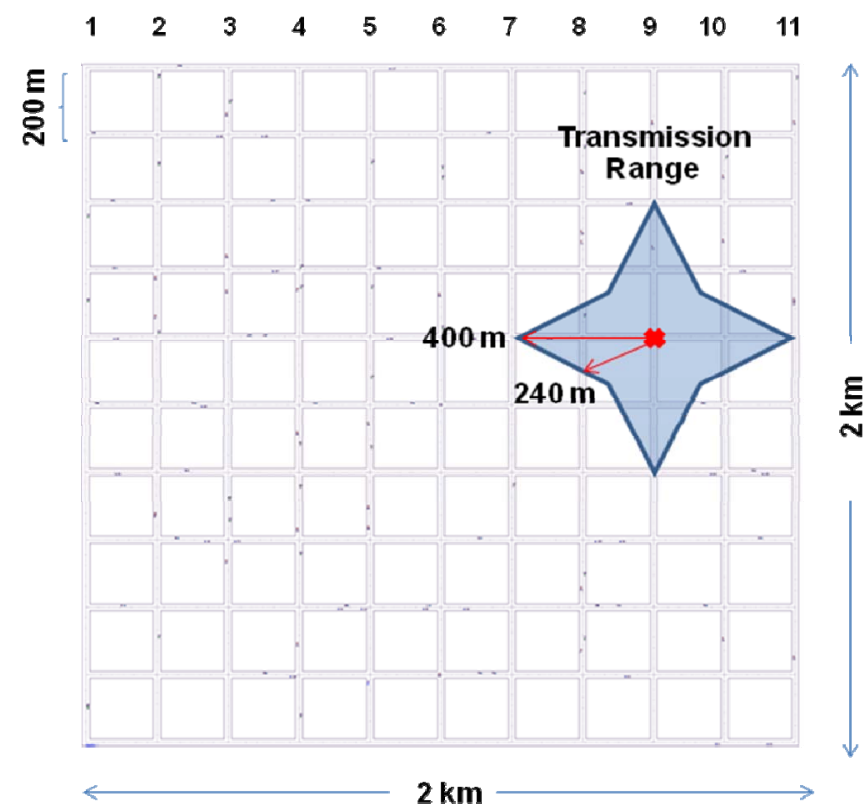

Fig. 1. Network topology with Manhattan Grid Structure

- Network Topology: Topology constructed with 200m x $200 \mathrm{~m}$ blocks over a $2 \mathrm{~km} \times 2 \mathrm{~km}$ area. Two-ways roads are used with one lane at each way. Vehicles distributed randomly over the roads avoiding collisions with one another.

- Movement Pattern: Vehicles move at predetermined constant speed. Measurements have been made at different vehicle speeds in order to observe the impact of vehicle speeds to the network performance. Vehicles approaching to the junctions either change their directions randomly to left or right or move on the same direction.

- Packet Types and Messaging Methods: Two types of packets are generally used in Vehicular Ad Hoc Networks [9]. First one is single-hop broadcast type (beaconing) packets and the second one is multi-hop unicast (may define a certain area) packets. Beaconing packets containing routine traffic information comprised of location, speed, direction of the vehicle are forwarded as single hop messages. Emergency warning messages such as accident notification and call for help and application specific messages used by commercial applications requiring peer to peer communications use the second type (unicast, multi-hop) packets. In simulations, routine traffic message are broadcasted with 3 second intervals as single hop packets. For unicast peer-to-peer communication, source/destination pairs are chosen randomly and peer-to-peer data is forwarded with a load ratio of 1 event/minute per vehicle. Peer-to-peer data is sent as 5 successive unicast data packets of which make a load ratio of 5 packets/minute per vehicle in addition to the beaconing packets. All types of packets are UDP packets. We assumed that each node has knowledge of its neighborhood. As our main goal is to determine the effect of lifetime on the packet delivery ratio, in experiments, buffer size is kept finite but large enough to allow packets to be queued. Therefore the packet drops due to insufficient buffer size are prevented to affect the packet delivery ratio.

- Communication Model: $400 \mathrm{~m}$ transmission range [14], [15] with free space communication model is used for the vehicles. This transmission range value is the communication range which is accepted according to the DSRC standards [16]. As the signal propagation is affected from structures such as buildings, the communication range for the vehicles which are not on the same road has been accepted as $240 \mathrm{~m}$ considering the block diagonal length.

- Simulation Speed: Results are obtained after 10 runs. Each simulation precedes with by 1 millisecond intervals and ends after 180 seconds.

- Measurement Factors: Measurements are observed for networks with different densities $(40 \%, 60 \%, 80 \%$, $100 \%$ ) and with different vehicle speeds. Some of the simulation system parameters are summarized in Table I.

TABLE I

SIMULATION PARAMETERS

\begin{tabular}{|l|l|}
\hline \multicolumn{1}{|c|}{ Parameter } & \multicolumn{1}{c|}{ Value } \\
\hline Simulation Area & $2 \mathrm{~km} \times 2 \mathrm{~km}$ \\
\hline Number of Vehicles & $70,96,120$ vehicles \\
\hline Transmission Range & $400 \mathrm{~m}, 240 \mathrm{~m}$ \\
\hline Simulation Duration & $180000 \mathrm{msec}$ \\
\hline Unit Time Process & $1 \mathrm{msec}$ \\
\hline Block Size & $200 \mathrm{~m} \times 200 \mathrm{~m}$ \\
\hline
\end{tabular}




\section{B. Simulation Results}

In the first experiment, we evaluate the performance of partially connected network in terms of packet delivery ratio. If the nodes which are about to transmit a packet cannot find a next addressable node to forward the packet due to the partial connectivity, they will drop the packet in NDTN. Since delaying packets on transmissions and extending the packet lifetime will reduce packet losses, effects of packet lifetime to packet delivery ratio is examined in this experiment.

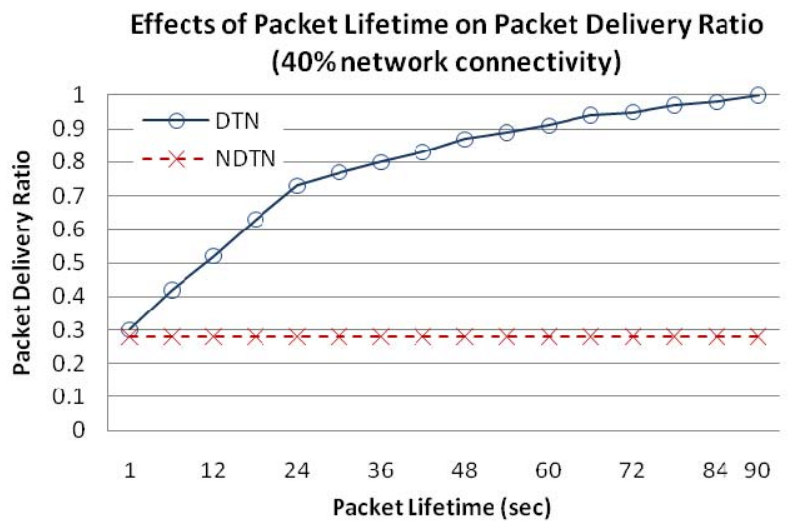

Fig. 2. Effects of Lifetime on Packet Delivery Ratio. Network Connectivity is $40 \%$ and there are totally 70 vehicles in the network.

Fig. 2 shows the effects of packet lifetime to packet delivery ratio in a network where the network connectivity is $40 \%$ and vehicle speed is $60 \mathrm{~km} / \mathrm{h}$. It is clearly seen that increasing the packet lifetime in Delay Tolerant Networks increases the packet delivery ratio reaching the highest value $(100 \%)$ at second 90 which is also the ratio for a full connected network. The payoff in DTNs is the delay $(90 \mathrm{sec})$ encountered due to $40 \%$ partial connectivity. If it would be a full connected network, packets would arrive to destination in less than one second (about $0.1 \mathrm{sec}$ ). In Non-Delay Tolerant Network, packets are also delivered in less than one second, but increasing the lifetime does not have any positive effect on successful packet delivery. Packet delivery ratio remains constant at $28 \%$.

Nodes in NDTNs drop the packet regardless of packet lifetime if they cannot find a next addressable node to forward the packet. The extension of the packet lifetime in NDTNs (the lifetime that is determined for a full connected network) does not increase the data delivery ratio in partially connected networks. On the other hand, nodes in DTNs carry the packet until they find a next addressable node. Node movements during their carry time causes to form new clusters enabling the nodes find appropriate nodes to forward the packet, thus enhance the packet delivery ratio.

Carrying packets in DTNs causes delayed packet delivery at destination nodes. Delay duration varies according to the network density. Therefore, effects of changing network connectivity to the packet lifetime are examined. Network connectivity rates are shown in Table II. Fig. 3 shows the obtained results. In DTNs, maximum lifetime needed to deliver all packets is 90 seconds for $40 \%$ network connectivity and this value reduces to 48 seconds for $80 \%$ network connectivity. Increasing the network density in partially connected network (that is a transition from partial connectivity to the full connectivity) shortens the needed packet lifetime to deliver all packets with a packet delivery ratio $100 \%$. However, in NDTNs, even though the data delivery ratios change depending on the network connectivity rates, extending the packet lifetime does not have any positive effect on packet delivery ratio.

\section{Effects of Network Connectivity \& Packet Lifetime to} Packet Delivery Ratio

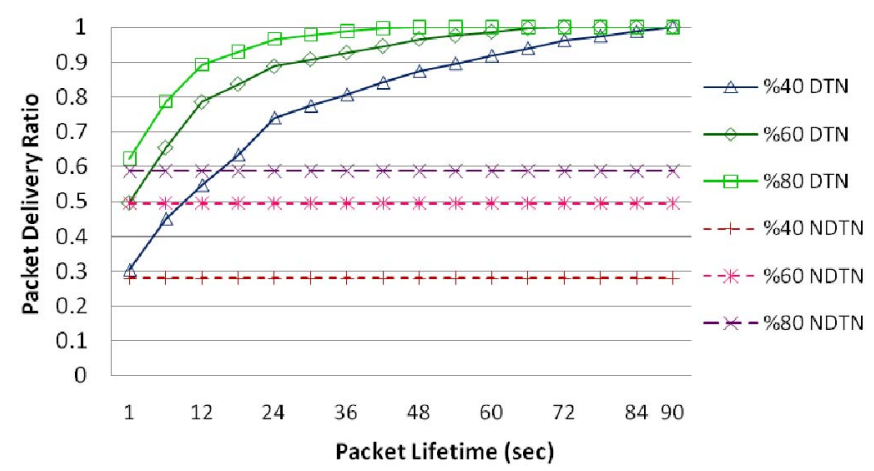

Fig. 3. Effects of network connectivity rate and packet lifetime to packet delivery ratio.

TABLE II

CONNECTIVITY RATES AND NUMBER OF VEHICLES

\begin{tabular}{|c|c|}
\hline Connectivity Rate & Number of Vehicles \\
\hline $40 \%$ & 70 vehicles $\left(18\right.$ vehicle $\left./ \mathrm{km}^{2}\right)$ \\
\hline $60 \%$ & 96 vehicles $\left(24\right.$ vehicle $\left./ \mathrm{km}^{2}\right)$ \\
\hline $80 \%$ & 120 vehicles $\left(30\right.$ vehicle $\left./ \mathrm{km}^{2}\right)$ \\
\hline $100 \%$ & 180 vehicles $\left(45\right.$ vehicle $\left./ \mathrm{km}^{2}\right)$ \\
\hline
\end{tabular}

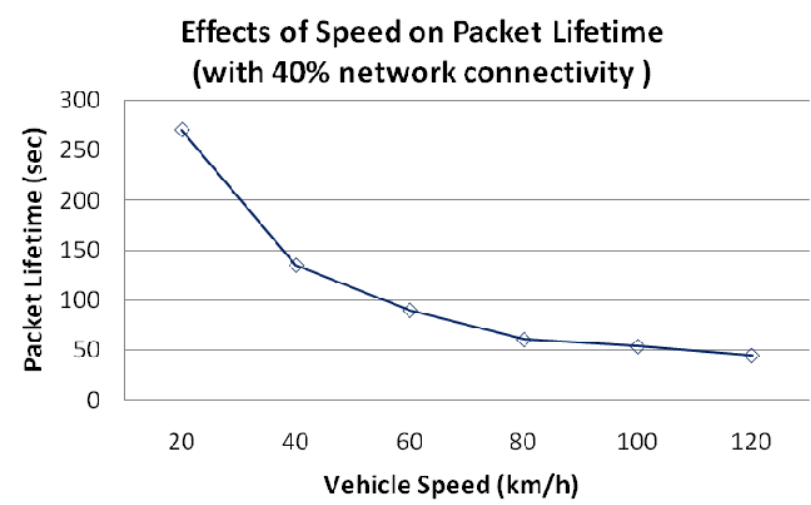

Fig. 4. Effects of speed change on packet lifetime to provide $100 \%$ packet delivery ratio.

As mentioned previously, in partially connected networks the packet delivery ratio increases as the vehicles move into other clusters forming new clusters. For a given network density, a vehicle needs to move through a definite distance to merge into new cluster to be able forward its packets. Then, the time interval needed for transition from one cluster to 
another one is related with the speed of nodes and the connectivity ratio of the network. Nodes' packet carrying time and the lifetime of packets within the network can be determined according to the speed of the vehicles. In a network with high speed vehicles, vehicles drive through to other clusters in a shorter time with respect to a network with low speed vehicles. This causes the vehicle interchange time between the clusters to be shorter in the former one. In this case, packet carry time at nodes and lifetime of the packets will also be shorter to ensure $100 \%$ packet delivery ratio. Fig. 4 shows the effects of varying vehicle speed to packet lifetime to sustain $100 \%$ packet delivery ratio. As the speed of vehicles increases, packet lifetime decreases. Fig. 3 and 4 can be used determine the lifetime of specific applications which are bounded on end-to-end delay. If determined lifetime exceeds the application's bounded delay, then it is a tradeoff either to accept the delay or to admit dropping packets with respect to current network parameters.

TABLE III

Delay Rates of WiRELESS TECHNOLOGIES [16]

\begin{tabular}{|l|l|}
\hline Wireless Technology & Delay Time \\
\hline Two-way Satellite & $60 \mathrm{sec}$ and over \\
\hline Terrestrial Digital Radio System & $10-20 \mathrm{sec}$ \\
\hline Satellite Digital Audio Radio System & $10-20 \mathrm{sec}$ \\
\hline IEEE 802.11 Wireless LAN & $3-5 \mathrm{sec}$ \\
\hline Digital Television & $10-30 \mathrm{sec}$ \\
\hline 2.5 - 3G PCS and Cellular Networks & $3-5 \mathrm{sec}$ \\
\hline
\end{tabular}

Delay payoff due to store-carry-forward mechanisms in delay tolerant networks may seem high. However, compared to the other communication technologies as shown in Table III, it is at a moderate level. Values in Table III [16] can be considered as the upper bound for a successful message delivery, since they are also very high. Considering today's technologies, end-to-end delay times for the wireless technologies given in Table III will be much less than these values. These values are given to show that delay encountered in store-carry-forward approach remains at an acceptable value. However, applications sensitivity on delay determines the acceptable end-to-end delay. For partially connected networks, high data rate in DSRC provides a very short delay indeed compared to other wireless technologies presented in Table III.

Delay encountered in delay tolerant networks can also be reduced with a heterogeneous structure comprising of fixed infrastructure elements (e.g. roadside units, GSM infrastructure). However, the purpose of this study is to present approaches for more reliable communications that does not depend on any fixed infrastructure. This approach enables to be used at any time even when fixed infrastructure components don't operate. Another benefit of this approach is related with cost. Compared to the alternative communication methods shown in Table III, Vehicular Ad hoc Networks provides high data rates with low communication cost.

\section{CONCLUSION AND FUtURE WORK}

In vehicular networks, data delivery is affected by the connectivity of the network. Data delivery ratio decreases as the connectivity of network decreases. Packet drops arising from the connection loss or route breakages in data delivery can be decreased by store-carry-forward method. Key to this approach is determination of carry time for vehicles and lifetime of the packet. In this study, effects of carry time of packets by vehicles and packet's lifetime on packet delivery ratio in the Sparse Vehicular Networks are examined. At the same time, effects of vehicle speeds and network density on the packet lifetime are examined. In partially connected networks, it is shown that extending packet lifetime increases packet delivery ratio with a delay payoff due to store-carryforward method. Depending on the network density and vehicle speed, lifetime of packets is determined. This is the first study for determination of packet lifetime in Sparse Vehicular Networks. It gives a better knowledge of the effects of the store-carry-forward mechanism on the end-to-end packet delivery ratio in sparse vehicular networks. This study also contributes to the fulfillment of the Quality of Service requirements of applications. As the required Quality of Service will vary with regard to applications, the studies to meet these Quality of Service requirements of different applications with an acceptable delay tolerance by determining the appropriate lifetime forms the future studies.

\section{REFERENCES}

[1] (2010) DSRC Website at Berkeley University. [Online] Available: http://path.berkeley.edu/dsrc/

[2] O. K. Tonguz, N.Wisitpongphan, J. S. Parikh, F. Bai, P. Mudalige, and V. K. Sadekar,"On the Broadcast Storm Problem in Ad hocWireless Networks" International Conference on Broadband Communications, Networks and Systems, Oct. 2006, pp.1-11.

[3] K. C. Lee, U. Lee, M. Gerla. "Survey of Routing Protocols in Vehicular Ad Hoc Networks", Advances in Vehicular Ad-Hoc Networks: Developments and Challenges, IGI Global, Oct, 2009.

[4] Y.-W. Lin, Y.-S. Chen, S.-L. Lee, "Routing Protocols in Vehicular Ah Hoc Networks: A Survey and Future Perspectives," Journal of Information Science and Engineering, 2009.

[5] A. Vahdat, D. Becker. "Epidemic Routing for Partially-Connected Ad Hoc Networks", Duke University, Technical Report CS-200006, April 2000.

[6] T. Spyropoulos, K. Psounis, C. S. Raghavendra. "Spray and Wait: Efficient routing scheme for intermittently connected mobile networks", ACM SIGCOMM workshop on Delay Tolerant Networking (WDTN), 2005.

[7] J. LeBrun, C. N. Chuah, D. Ghosal. "Knowledge- Based Opportunistic Forwarding in Vehicular Wireless Ad Hoc Networks", IEEE VTC, Spring 2005.

[8] J. Kurhinen, J. Janatuinen. "Delay Tolerant Routing In Sparse Vehicular Ad Hoc Networks", Acta Electrotechnica et Informatica, Vol. 8, No. 3, 2008, 7-13

[9] A. Skordylis, N. Trigoni. "Delay-bounded Routing in Vehicular Adhoc Networks", MobiHoc'08, May 2008.

[10] K. C. Lee, A. Piechowicz, M. Gerla, A. Tiwari, A. Ganguli, D. Krzysiak. "Delay Tolerant Mobility Aware Routing/Mobility Dissemination Protocol for the Airborne Network," IEEE MILCOM 2009, Boston, MA, October 2009.

[11] A. Tiwari, A. Ganguli, A. Sampath, D. S. Anderson, B.-h. Shen, N. Krishnamurthi, J. Yadegar, M. Gerla, D. Krzysiak. "Mobility Aware 
Routing for the Airborne Network Backbone", IEEE MILCOM 2008, San Diego, CA, October 2008.

[12] (2010) ETSI, Universal Mobile Telecommunication System (UMTS), "Selection procedures for the choice of radio transmission Technologies of the UMTS", UMTS 30.03 Version 3.2.0, 1998-04. [Online]. Available:

http://www.3gpp.org/ftp/Specs/html-info/3003U.htm.

[13] N. Wisitpongphan, F. Bai, P. Mudalige, V. Sadekar, and O. Tonguz. "Routing in Sparse Vehicular Ad Hoc Wireless Networks", IEEE Journal on Selected Areas in Communications, Vol. 25, No. 8, October 2007.

[14] J. Nzouonta, N. Rajgure, G. Wang, and C. Borcea. "VANET Routing on City Roads using Real-Time Vehicular Traffic Information", IEEE Transactions on Vehicular Technology, Vol. 58, No. 7, 2009.

[15] N. Eude, B. Ducourthial, and M. Shawky. "Enhancing NS-2 simulator for high mobility ad hoc networks in car-to-car communication", 7th IFIP International Conference on Mobile and Wireless Communications Networks, Marrakech, Morocco, September 2005.

[16] Vehicle Safety Communications Project Task 3 Final Report, "Identify intelligent vehicle safety applications enabled by dsrc, dot hs 809 859", NHTSA, March 2005. http://www-nrd.nhtsa.dot.gov

[17] R. Shirani, F. Hendessi. "A Markov Chain Model for Evaluating Performance of Store-Carry-Forward Procedure in VANETs", IEEE-ICCS, 2008. 